\title{
A PUSHING-PULLING METHOD: NEW PROOFS OF INTERSECTION THEOREMS
}

\author{
RUDOLF AHLSWEDE and LEVON H. KHACHATRIAN
}

Received March 24, 1997

\section{Introduction}

$\mathbb{N}$ denotes the set of positive integers. For $i, j \in \mathbb{N}, i<j$, the set $\{i, i+1, \ldots, j\}$ is abbreviated as $[i, j]$ and we write $[n]$ for $[1, n]$. For $k, n \in \mathbb{N}, k \leq n$, we set

$$
2^{[n]}=\{F: F \subset[n]\}, \quad\left(\begin{array}{c}
{[n]} \\
k
\end{array}\right)=\left\{F \in 2^{[n]}:|F|=k\right\} .
$$

A system of sets $\mathcal{A} \subset 2^{[n]}$ is called $t$-intersecting, if $\left|A_{1} \cap A_{2}\right| \geq t$ for all $A_{1}, A_{2} \in \mathcal{A}$ (for $t=1$, we call $\mathcal{A}$ just intersecting). $\mathcal{I}(n, t)$ denotes the set of all such systems and for the systems restricted to $k$-element sets we introduce

$$
\mathcal{I}(n, k, t)=\left\{\mathcal{B} \in \mathcal{I}(n, t): \mathcal{B} \subset\left(\begin{array}{c}
{[n]} \\
k
\end{array}\right)\right\} .
$$

We are interested in the functions

$$
M(n, t)=\max _{\mathcal{A} \in \mathcal{I}(n, t)}|\mathcal{A}| \text { and } M(n, k, t)=\max _{\mathcal{A} \in \mathcal{I}(n, k, t)}|\mathcal{A}| .
$$

For their analysis we introduce the following sets:

$$
\mathcal{K}(n, t)=\left\{A \in 2^{[n]}:|A| \geq \frac{n+t}{2}\right\}=\bigcup_{i=\frac{n+t}{2}}^{n}\left(\begin{array}{c}
{[n]} \\
i
\end{array}\right) \text { if } 2 \mid(n+t)
$$

and

$$
\mathcal{F}_{i}=\left\{F \in\left(\begin{array}{c}
{[n]} \\
k
\end{array}\right):|F \cap[t+2 i]| \geq t+i\right\} \text { for } 0 \leq i \leq k-t .
$$

Mathematics Subject Classification (1991): 05D05 
There is a well-known result of Katona, which determines the exact value of $M(n, t)$ for all $n, t$.

Theorem Ka. [3]

$$
M(n, t)= \begin{cases}|\mathcal{K}(n, t)| & \text { if } 2 \mid(n+t) \\ 2 \cdot|\mathcal{K}(n-1, t)| & \text { if } 2 \nmid(n+t) .\end{cases}
$$

Moreover, in the case $2 \mid(n+t), t>1$, the optimal configuration is unique, while in the case $2 \nmid(n+t)$ it is unique up to permutations of the ground set $[n]$.

The proof of this Theorem in [3] is essentially based on a result concerning shadows of $t$-intersecting systems.

Recently we proved a long-standing conjecture concerning the function $M(n, k, t)$.

Theorem AK. [1] For $1 \leq t \leq k \leq n, n>2 k-t$ with

(i)

$$
(k-t+1)\left(2+\frac{t-1}{r+1}\right)<n<(k-t+1)\left(2+\frac{t-1}{r}\right) \text { for some } r \in \mathbb{N} \cup\{0\}
$$

we have

$$
M(n, k, t)=\left|\mathcal{F}_{r}\right|
$$

and $\mathcal{F}_{r}$ is - up to permutations - the unique optimum. (By convention $\frac{t-1}{r}=\infty$ for $r=0$.)

$$
(k-t+1)\left(2+\frac{t-1}{r+1}\right)=n \text { for } r \in \mathbb{N} \cup\{0\} \text { and } t>1
$$

we have

$$
M(n, k, t)=\left|\mathcal{F}_{r}\right|=\left|\mathcal{F}_{r+1}\right|
$$

and an optimal system equals - up to permutations - either $\mathcal{F}_{r}$ or $\mathcal{F}_{r+1}$.

For the proof we introduced the seemingly basic notion of - what we called"generating sets".

In the present paper we provide a new compression method, which leads to new proofs for both, Theorem Ka and Theorem AK.

The new method, in some sense, can be considered as a "dual" to the method of "generating sets".

Independent of the new method, in the last section, we derive Theorem Ka from Theorem AK by a simple analytical approach. 


\section{Left compressed sets and an auxiliary result}

We begin with well-known notions.

Definition 2.1. For $A_{1}=\left\{i_{1}, i_{2}, \ldots, i_{s}\right\} \in\left(\begin{array}{c}{[n]} \\ s\end{array}\right), i_{1}<i_{2}<\ldots<i_{s}$, and $A_{2}=$ $\left\{j_{1}, j_{2}, \ldots, j_{s}\right\} \in\left(\begin{array}{c}{[n]} \\ s\end{array}\right), j_{1}<j_{2}<\ldots<j_{s}$, we write

$$
A_{1} \prec A_{2} \text { if } i_{\ell} \leq j_{\ell} \text { for all } 1 \leq \ell \leq s,
$$

that is, $A_{1}$ can be obtained from $A_{2}$ by left-pushing. Furthermore, let $\mathcal{L}\left(A_{2}\right)$ be the set of all sets obtained this way from $A_{2}$. Also set

$$
\mathcal{L}(\mathcal{A})=\bigcup_{A \in \mathcal{A}} \mathcal{L}(A)
$$

Definition 2.2. $\mathcal{A} \subset 2^{[n]}$ is said to be left compressed if $\mathcal{A}=\mathcal{L}(\mathcal{A})$.

Definition 2.3. We denote by $L \mathcal{I}(n, t) \subset \mathcal{I}(n, t),(\operatorname{resp} . \quad \mathcal{L} \mathcal{I}(n, k, t) \subset \mathcal{I}(n, k, t))$ the set of all left compressed systems belonging to $\mathcal{I}(n, t)$ (resp. to $\mathcal{I}(n, k, t)$ ).

It is well known and it easily follows with the shifting technique of [2] that

$$
M(n, t)=\max _{\mathcal{A} \in \mathcal{I}(n, t)}|\mathcal{A}|=\max _{\mathcal{A} \in L \mathcal{I}(n, t)}|\mathcal{A}|
$$

and

$$
M(n, k, t)=\max _{\mathcal{A} \in \mathcal{I}(n, k, t)}|\mathcal{A}|=\max _{\mathcal{A} \in L \mathcal{I}(n, k, t)}|\mathcal{A}| .
$$

Definition 2.4. For any $B \in 2^{[n]}$ we define the upset $\mathcal{U}(B)=\left\{B^{\prime} \in 2^{[n]}: B \subset B^{\prime}\right\}$.

More generally, for $\mathcal{B} \subset 2^{[n]}$ we define the upset

$$
\mathcal{U}(\mathcal{B})=\bigcup_{B \in \mathcal{B}} \mathcal{U}(B)
$$

Clearly, every optimal set $\mathcal{A} \in \mathcal{L}(n, t)$ is an upset.

The next result shows that in the investigation of the function $M(n, t)$ we can concentrate on the case $2 \mid(n+t)$.

Lemma 1. Let $2 \nmid(n+t)$. Then

$$
M(n, t)=2 \cdot M(n-1, t) .
$$

Proof. Clearly $M(n, t) \geq 2 \cdot M(n-1, t)$. Let us prove the opposite direction. Let $\mathcal{A} \in \mathcal{L} \mathcal{I}(n, t),|\mathcal{A}|=M(n, t), 2 \nmid(n+t)$, and let

$$
\mathcal{A}_{n}=\{A \in \mathcal{A}: n \in A, \text { but }(A \backslash\{n\}) \notin \mathcal{A}\} .
$$


If $\mathcal{A}_{n}=\emptyset$, then obviously $\left|A_{1} \cap A_{2} \cap[1, n-1]\right| \geq t$ for all $A_{1}, A_{2} \in \mathcal{A}$, and hence $M(n, t) \leq 2 M(n-1, t)$.

Assuming $\mathcal{A}_{n} \neq \emptyset$, consider the following partition: $\mathcal{A}_{n}=\mathcal{A}_{n}^{1} \dot{\cup} \mathcal{A}_{n}^{2}$, where $\mathcal{A}_{n}^{1}=\left\{A \in \mathcal{A}_{n}:|A| \geq \frac{n+t+1}{2}\right\}$ and $\mathcal{A}_{n}^{2}=\left\{A \in \mathcal{A}_{n}:|A| \leq \frac{n+t-1}{2}\right\}$.

We observe that for any $A_{1}, A_{2} \in \mathcal{A}_{n}^{1} \quad\left|A_{1} \cap A_{2}\right| \geq t+1$ holds, and the same is true for any $A_{3}, A_{4} \in \mathcal{A}_{n}^{2}$, because $\mathcal{A}$ is left-compressed, $\mathcal{A} \in \mathcal{I}(n, t)$, and $n \in A_{3} \cap A_{4}$.

Therefore, the sets

$$
\mathcal{B}_{n}^{i}=\left\{B \in 2^{[n]}: n \notin B, \text { but } B \cup\{n\} \in \mathcal{A}_{n}^{i}\right\}(i=1,2)
$$

are $t$-intersecting. Consequently we have

$$
\mathcal{C}_{1}=\left(\mathcal{A} \backslash \mathcal{A}_{n}^{1}\right) \cup \mathcal{B}_{n}^{2} \in \mathcal{I}(n, t), \quad \mathcal{C}_{2}=\left(\mathcal{A} \backslash \mathcal{A}_{n}^{2}\right) \cup \mathcal{B}_{n}^{1} \in \mathcal{I}(n, t) .
$$

Since $\mathcal{B}_{n}^{i} \notin \mathcal{A}(i=1,2)$ by definition of $\mathcal{A}_{n}$ and $\left|\mathcal{B}_{n}^{i}\right|=\left|\mathcal{A}_{n}^{i}\right|(i=1,2)$, clearly

$$
\max _{i=1,2}\left|\mathcal{C}_{i}\right| \geq|\mathcal{A}|=M(n, t) .
$$

Moreover, with every element $C \in \mathcal{C}$ with $n \in C$ we have also that $C^{\prime \prime}=C \backslash$ $\{n\} \in \mathcal{C}_{1}$. Of course, the same is true for the set $\mathcal{C}_{2}$.

Therefore, for all $C_{1}, C_{2} \in \mathcal{C}_{1}$ (similarly for $\mathcal{C}_{2}$ ) we have $\mid C_{1} \cap C_{2} \cap[1, n-1] \geq t$ and hence

$$
2 \cdot M(n-1, t) \geq \max _{i=1,2}\left|\mathcal{C}_{i}\right| \geq\left|\mathcal{A}_{i}\right|=M(n, t) .
$$

This proves the lemma.

For a set $\mathcal{A} \subset 2^{[n]}$ and $1 \leq i, j \leq n$ we denote by $\mathcal{A}_{i, j}$ the set which is obtained from the set $\mathcal{A}$ by exchanging the coordinates $i, j$ in every $A \in \mathcal{A}$.

Suppose $\mathcal{A} \in L \mathcal{I}(n, t)$, (resp. $\mathcal{A} \in \mathcal{L} \mathcal{I}(n, k, t)$ ) and $\mathcal{A}$ is not right-compressed. Let $\ell<n$ be the biggest integer, such that $\mathcal{A}$ is invariant under exchange operations in $[1, \ell]$, i.e.

$$
\mathcal{A}=\mathcal{A}_{i, j} \text { for all } 1 \leq i, j \leq \ell \text { but } \mathcal{A} \neq \mathcal{A}_{i, \ell+1} \text { for some } 1 \leq i \leq \ell .
$$

Moreover let

$$
\mathcal{A}^{\prime}=\left\{A \in \mathcal{A}: A_{i, \ell+1} \notin \mathcal{A} \text { for some } 1 \leq i \leq \ell\right\} .
$$

We need the following easy, but important

Lemma 2. Let $\mathcal{A}$ and $\mathcal{A}^{\prime}$ be the sets which are defined just above. Then

(i) $\ell+1 \notin A$ for all $A \in \mathcal{A}^{\prime}$.

(ii) Let $A \in \mathcal{A}^{\prime}$ and $j \in A, 1 \leq j \leq \ell$, then we have $A_{j, \ell+1} \notin \mathcal{A}$. 
(iii) Let $A \in \mathcal{A}^{\prime}, A=B \cup C$, where $B=A \cap[1, \ell], C=A \cap[\ell+1, n]$, then we have $B^{\prime} \cup C \in \mathcal{A}^{\prime}$ for every $B^{\prime} \subset[1, \ell]$ with $\left|B^{\prime}\right|=|B|$.

(iv) Let $A \in \mathcal{A}^{\prime}$ and $D \in \mathcal{A} \backslash \mathcal{A}^{\prime}$, then we have

$$
\left|A_{i, \ell+1} \cap D\right| \geq t \text { for all } 1 \leq i \leq \ell .
$$

(v) Let $A_{1}, A_{2} \in \mathcal{A}^{\prime}, B_{i}=A_{i} \cap[1, \ell](i=1,2)$ and suppose that $\left|B_{1}\right|+\left|B_{2}\right| \neq \ell+t$, then we have $\left|A_{1} \cap A_{2}\right| \geq t+1$.

Proof. The statement immediately follows from the left-compressedness of $\mathcal{A}$, the definition of $\mathcal{A}^{\prime}$ and the maximality of $\ell$.

We state also the following (almost trivial) fact.

Lemma 3. Let $\mathcal{B} \subset 2^{[n]}$ be a set system, such that

$$
B \in \mathcal{B} \rightarrow \bar{B} \in \mathcal{B}, \text { where } \bar{B}=[1, n] \backslash B
$$

Then every maximal (saturated) intersecting $\mathcal{B}^{\prime} \subset \mathcal{B}$ has cardinality $\frac{|\mathcal{B}|}{2}$. Here maximal (saturated) means, that we cannot add another element to the set without violating the intersecting property.

The following statement is obvious.

Lemma 4. Let $2 \mid(n+t)$, Then (i) and (ii) are equivalent:

(i) The unique optimal set $\mathcal{A}$ with $|\mathcal{A}|=M(n, t)$ is $\mathcal{A}=\mathcal{K}(n, t)$.

(ii) Every optimal set is left-compressed and at the same time it is rightcompressed.

\section{Proof of Theorem AK}

The main auxiliary result, which essentially proves the theorem, is the following.

Lemma 5. Let $\mathcal{A} \subset L \mathcal{I}(n, k, t),|\mathcal{A}|=M(n, k, t), n>2 k-t$ and

$$
n<(k-t+1)\left(2+\frac{t-1}{r}\right) .
$$

Then $\mathcal{A}$ is invariant under exchange operations in $[1, t+2 r]$, i.e. $\mathcal{A}_{i, j}=\mathcal{A}$ for all $1 \leq i, j \leq t+2 r$. (By convention $\frac{t-1}{0}=\infty$ for $r=0$.)

Before we give a proof let us compare this lemma, with its dual, Lemma 6 [1]. In [1] this lemma was stated in the language of generated sets, here we give an equivalent formulation. 
Lemma 6. [1] Let $\mathcal{A} \subset L \mathcal{I}(n, k, t),|\mathcal{A}|=M(n, k, t), n>2 k-t$, and

$$
(k-t+1)\left(2+\frac{t-1}{r+1}\right)<n .
$$

Then $\left|A_{1} \cap A_{2} \cap[1, t+2 r]\right| \geq t$ for all $A_{1}, A_{2} \in \mathcal{A}$ or, equivalently, $\mathcal{A}$ is invariant under exchange operations in $[t+2 r+1, n]$.

It is easily seen, that the combination of the lemmas immediately proves Theorem AK, at least the first case (1.4).

Proof of Lemma 5. We can assume that $t \geq 2$, because in the case $t=1$ the conditions (3.1) and $n>2 k-t$ are incompatable. Assume the opposite and let $\ell<(t+2 r)$ be the biggest integer such that

$$
\begin{gathered}
\mathcal{A}_{i, j}=\mathcal{A} \text { for all } 1 \leq i, j \leq \ell, \text { but } \\
\mathcal{A}^{\prime}=\left\{A \in \mathcal{A}: A_{i, \ell+1} \notin \mathcal{A}, \text { for some } 1 \leq i \leq \ell\right\} \neq \emptyset .
\end{gathered}
$$

We are going to show that, under assumption (3.1) and $\ell<t+2 r$, there exists a $\mathcal{B} \in \mathcal{I}(n, k, t)$ with $|\mathcal{B}|>|\mathcal{A}|$, which is a contradiction.

For this we start with the partition of $\mathcal{A}^{\prime}$ :

$$
\mathcal{A}^{\prime}=\bigcup_{i=1}^{\ell} \mathcal{A}(i), \quad \text { where } \mathcal{A}(i)=\left\{A \in \mathcal{A}^{\prime}:|A \cap[1, \ell]|=i\right\} \text {. }
$$

Of course, some of the $\mathcal{A}(i)$ 's can be empty. In fact, it follows from Lemma 2 (v) and the maximality of $\mathcal{A}$ that $\mathcal{A}(i)=\emptyset$ for all $1 \leq i<t$. We will show that all the $\mathcal{A}(i)$ 's are empty. Suppose $\mathcal{A}(i) \neq \emptyset$ for some $i, t \leq i \leq \ell$.

From Lemma 2 (iii) we know that

$$
|\mathcal{A}(i)|=\left(\begin{array}{l}
\ell \\
i
\end{array}\right) \cdot\left|\mathcal{A}^{*}(i)\right|
$$

where

$$
\mathcal{A}^{*}(i)=\{A \cap[\ell+2, n]: A \in \mathcal{A}(i)\}
$$

We remind the reader that $\ell+1 \notin A$ for all $A \in \mathcal{A}^{\prime}$ (see Lemma 2 (i)).

Let us note that in the case $n=\ell+1$ we have $\mathcal{A}^{*}(i)=\{\emptyset\}$, and $\left|\mathcal{A}^{*}(i)\right|=1$.

Now we consider the set

$$
\mathcal{B}(i)=\left\{B:|B \cap[1, \ell]|=i-1, \ell+1 \in B,(B \cap[\ell+2, n]) \in \mathcal{A}^{*}(i)\right\} .
$$




\section{Clearly}

$$
|\mathcal{B}(i)|=\left(\begin{array}{c}
\ell \\
i-1
\end{array}\right) \cdot\left|\mathcal{A}^{*}(i)\right| \text { and } \mathcal{B}(i) \cap \mathcal{A}=\emptyset \text { by Lemma } 2 \text { (ii). }
$$

With $\mathcal{A}(i)$ and $\mathcal{B}(i)$ we consider also the sets $\mathcal{A}(\ell+t-i)$ and $\mathcal{B}(\ell+t-i)$. Similar to $(3.3)$, (3.5) we have

$$
|\mathcal{A}(\ell+t-i)|=\left(\begin{array}{c}
\ell \\
\ell+t-i
\end{array}\right) \cdot\left|\mathcal{A}^{*}(\ell+t-i)\right|
$$

and

$$
|\mathcal{B}(\ell+t-i)|=\left(\begin{array}{c}
\ell \\
\ell+t-i-1
\end{array}\right) \cdot\left|\mathcal{A}^{*}(\ell+t-i)\right| .
$$

We distinguish two cases: a) $i \neq \ell+t-i$, b) $i=\ell+t-i$.

Case a) $i \neq \ell+t-i$ :

From Lemma $2(\mathrm{v})$ it follows:

For $B \in \mathcal{B}(i), A \in \mathcal{A}(j)$ with $i+j \neq \ell+t$ we have $|B \cap A| \geq t$. Hence, using this and Lemma 2 (iv), we have

$$
\begin{aligned}
& \mathcal{H}_{1}=((\mathcal{A} \backslash \mathcal{A}(\ell+t-i)) \cup \mathcal{B}(i)) \in \mathcal{I}(n, k, t) \text { and } \\
& \mathcal{H}_{2}=((\mathcal{A} \backslash \mathcal{A}(i)) \cup \mathcal{B}(\ell+t-i)) \in \mathcal{I}(n, k, t) .
\end{aligned}
$$

Let us show that

$$
\max \left\{\left|\mathcal{H}_{1}\right|,\left|\mathcal{H}_{2}\right|\right\}>|\mathcal{A}|=M(n, k, t),
$$

which will be a contradiction.

Negation of (3.8) in conjunction with (3.3), (3.5)-(3.7) means that

$$
\begin{gathered}
\left(\begin{array}{c}
\ell \\
i-1
\end{array}\right) \cdot\left|\mathcal{A}^{*}(i)\right| \leq\left(\begin{array}{c}
\ell \\
\ell+t-i
\end{array}\right) \cdot\left|\mathcal{A}^{*}(\ell+t-i)\right|, \\
\left(\begin{array}{c}
\ell \\
\ell+t-i-1
\end{array}\right) \cdot\left|\mathcal{A}^{*}(\ell+t-i)\right| \leq\left(\begin{array}{l}
\ell \\
i
\end{array}\right) \cdot\left|\mathcal{A}^{*}(i)\right| .
\end{gathered}
$$

Since we have assumed $\mathcal{A}(i) \neq \emptyset$, then clearly $\mathcal{A}(\ell+t-i) \neq \emptyset$ as well, because otherwise the first inequality of (3.9) is false.

However (3.9) implies

$$
i(\ell+t-i) \leq(\ell-i+1)(i+1-t),
$$

which is false, because $t \geq 2$ and consequently

$$
i>i+1-t, \ell+t-i>\ell-i+1 \text {. }
$$


Hence $\mathcal{A}(i)=\emptyset$ for all $i \neq \ell+t-i$.

Remark. Let us note, that we did not use the condition (3.1) in the Lemma.

Case b) $i=\ell+t-i$ or $i=\frac{\ell+t}{2}$ :

Here necessarily $2 \mid(\ell+t)$ and hence $\ell+2 \leq n$. Therefore, if $\mathcal{A}\left(\frac{\ell+t}{2}\right) \neq \emptyset$, then also $\mathcal{A}^{*}\left(\frac{\ell+t}{2}\right) \neq \emptyset$.

We have

$$
\left|\mathcal{A}\left(\frac{\ell+t}{2}\right)\right|=\left(\begin{array}{c}
\ell \\
\frac{\ell+t}{2}
\end{array}\right) \cdot\left|\mathcal{A}^{*}\left(\frac{\ell+t}{2}\right)\right| \quad(\text { see }(3.3))
$$

and any $A \in \mathcal{A}\left(\frac{\ell+t}{2}\right)$ can be written in the form $A=B \cup \dot{ } C$, where

$$
B=(A \cap[1, \ell]) \in\left(\begin{array}{c}
{[\ell]} \\
\frac{\ell+t}{2}
\end{array}\right), C=(A \cap[\ell+2, n]) \in \mathcal{A}^{*}\left(\frac{\ell+t}{2}\right),
$$

and $|C|=k-\frac{\ell+t}{2}$, since $A \in\left(\begin{array}{c}{[n]} \\ k\end{array}\right)$ and $\ell+1 \notin A$.

By the pigeon hole principle, there exists an element $d$ of $[\ell+2, n]$ and a subset $\mathcal{D} \subset \mathcal{A}^{*}\left(\frac{\ell+t}{2}\right)$ with $d \in D$ for all $D$ from $\mathcal{D}$ and

$$
|\mathcal{D}| \geq\left|\mathcal{A}^{*}\left(\frac{\ell+t}{2}\right)\right| \cdot \frac{k-\frac{\ell+t}{2}}{n-\ell-1}
$$

Now let

$$
\begin{aligned}
\mathcal{A}\left(\frac{\ell+t}{2}\right) & =\mathcal{A}_{1}\left(\frac{\ell+t}{2}\right) \dot{\cup} \mathcal{A}_{2}\left(\frac{\ell+t}{2}\right), \text { where } \\
\mathcal{A}_{1}\left(\frac{\ell+t}{2}\right) & =\left\{A \in \mathcal{A}\left(\frac{\ell+t}{2}\right):(A \cap[\ell+2, n]) \in \mathcal{D}\right\} \text { and } \\
\mathcal{A}_{2}\left(\frac{\ell+t}{2}\right) & =\mathcal{A}\left(\frac{\ell+t}{2}\right) \backslash \mathcal{A}_{1}\left(\frac{\ell+t}{2}\right) .
\end{aligned}
$$

Finally we consider the set

$$
\mathcal{H}=\left(\mathcal{A} \backslash \mathcal{A}_{2}\left(\frac{\ell+t}{2}\right)\right) \cup G
$$

where

$$
G=\left\{B \in\left(\begin{array}{c}
{[n]} \\
k
\end{array}\right):(\mathcal{B} \cap[1, \ell]) \in\left(\begin{array}{c}
{[\ell]} \\
\frac{\ell+t}{2}-1
\end{array}\right), \ell+1 \in B,(B \cap[\ell+2, n]) \in \mathcal{D}\right\} .
$$


By Lemma 2 (ii) $G \cap \mathcal{A}=\emptyset$, and it is easy to see that $\mathcal{H} \in \mathcal{I}(n, k, t)$.

Let us show that

$$
|\mathcal{H}|>|\mathcal{A}|
$$

under the conditions $(3.1), \ell<t+2 r$, and $2 \mid(\ell+t)$. The last two conditions imply

$$
\ell \leq t+2 r-2 \text {. }
$$

$$
|G|=\left(\begin{array}{c}
\ell+t \\
2
\end{array}-1\right) \cdot|\mathcal{D}| \text { and }\left|\mathcal{A}_{2}\left(\frac{t+\ell}{2}\right)\right|=\left(\begin{array}{c}
\ell \\
\frac{\ell+t}{2}
\end{array}\right) \cdot\left(\left|\mathcal{A}^{*}\left(\frac{t+\ell}{2}\right)\right|-|\mathcal{D}|\right) \text {, condition (3.11) }
$$

is equivalent to

$$
\left(\begin{array}{c}
\ell \\
\frac{\ell+t}{2}-1
\end{array}\right) \cdot|\mathcal{D}|>\left(\begin{array}{c}
\ell \\
\frac{\ell+t}{2}
\end{array}\right) \cdot\left(\left|\mathcal{A}^{*}\left(\frac{t+\ell}{2}\right)\right|-|\mathcal{D}|\right)
$$

and to

$$
\left(\begin{array}{c}
\ell+1 \\
\frac{\ell+t}{2}
\end{array}\right) \cdot|\mathcal{D}|>\left(\begin{array}{c}
\ell \\
\frac{\ell+t}{2}
\end{array}\right) \cdot\left|\mathcal{A}^{*}\left(\frac{t+\ell}{2}\right)\right|
$$

The inequality

$$
\left(\begin{array}{c}
\ell+1 \\
\frac{\ell+t}{2}
\end{array}\right) \cdot \frac{k-\frac{\ell+t}{2}}{n-\ell-1}>\left(\begin{array}{c}
\ell \\
\frac{\ell+t}{2}
\end{array}\right)
$$

is sufficient for (3.13) (see (3.10)).

After simplification (3.14) is equivalent to $(k-t+1)\left(2+\frac{2(t-1)}{\ell-t+2}\right)>n$, which is true, because $\ell \leq t+2 r-2$ (see (3.12)), and consequently

$$
(k-t+1)\left(2+\frac{2(t-1)}{\ell-t+2}\right) \geq(k-t+1)\left(2+\frac{t-1}{r}\right)>n, \text { which is (3.1). }
$$

The lemma is proved.

\section{Proof of Theorem AK.}

Case (i):

$$
(k-t+1)\left(2+\frac{t-1}{r+1}\right)<n<(k-t+1)\left(2+\frac{t-1}{r}\right) .
$$

Let $\mathcal{A} \in L \mathcal{I}(n, k, t)$ with $|\mathcal{A}|=M(n, k, t)$.

We know from Lemma 5 that $\mathcal{A}$ is invariant in $[1, t+2 r]$, hence obviously $k \geq t+r$, because otherwise we would have $A_{1}, A_{2} \in \mathcal{A}$ with $\left|A_{1} \cap A_{2}\right|<t$. 
Let $\overline{\mathcal{A}}=\{[1, n] \backslash A, A \in \mathcal{A}\}$ be the complemented set of $\mathcal{A}$. Clearly $\overline{\mathcal{A}}$ is rightcompressed, $\overline{\mathcal{A}} \in \mathcal{I}(n, n-k, n-2 k+t)$, and $|\mathcal{A}|=|\overline{\mathcal{A}}|=M(n, k, t)=M(n, n-k, n-2 k+t)$.

An easy calculation leads from (3.15) to

$$
\left(k^{\prime}-t^{\prime}+1\right)\left(2+\frac{t^{\prime}-1}{r^{\prime}+1}\right)<n<\left(k^{\prime}-t^{\prime}+1\right)\left(2+\frac{t^{\prime}-1}{r^{\prime}}\right)
$$

for $k^{\prime}=n-k, t^{\prime}=n-2 k+t$, and $r^{\prime}=k-t-r$ (by convention $\frac{t^{\prime}-1}{0}=\infty$ for $r^{\prime}=0$ ).

Using the dual version (with respect to right compressed sets) of Lemma 5 we get: $\overline{\mathcal{A}}$ is invariant in $\left[n-t^{\prime}-2 r^{\prime}+1, n\right]=[t+2 r+1, n]$.

Hence $\mathcal{A}$ is also invariant in $[t+2 r+1, n]$. Equivalently, since $\mathcal{A}$ is left compressed, $n>2 k-t$,

$$
\left|A_{1} \cap A_{2} \cap[1, t+2 r]\right| \geq t \text { for all } A_{1}, A_{2} \in \mathcal{A} .
$$

We know from Lemma 5 that

$$
\mathcal{A}_{i, j}=\mathcal{A} \text { for all } 1 \leq i, j \leq t+2 r .
$$

Clearly, the unique maximal set $\mathcal{A} \in L \mathcal{I}(n, k, t)$ is $\mathcal{A}=\mathcal{F}_{r}$.

Case (ii): $n=(k-t+1)\left(2+\frac{t-1}{r+1}\right)$.

Again, by considering the complemented set of $\mathcal{A}$, by the same approach we make the following conclusion, which is slightly different from (3.16):

$$
\left|A_{1} \cap A_{2} \cap[1, t+2 r+2]\right| \geq t \text { for all } A_{1}, A_{2} \in \mathcal{A} .
$$

It is easy to verify that combination of (3.17) with Lemma 5 gives exactly two optimal sets: either $\mathcal{A}=\mathcal{F}_{r}$ or $\mathcal{A}=\mathcal{F}_{r+1}$ and

$$
|\mathcal{A}|=\left|\mathcal{F}_{r}\right|=\left|\mathcal{F}_{r+1}\right| \text { for } n=(k-t+1)\left(2+\frac{t-1}{r+1}\right) \text {. }
$$

\section{First proof of Theorem Ka}

According to Lemma 1 we assume $2 \mid(n+t)$. From Lemma 4 it follows that in case $\mathcal{A} \in L \mathcal{I}(n, t),|\mathcal{A}|=M(n, t)$, we have to prove: $\mathcal{A}$ is also right-compressed. Assume the opposite, and let $\ell<n$ be the biggest integer such that $\mathcal{A}$ is invariant under exchange operations in $[1, \ell]$ and

$$
\mathcal{A}^{\prime}=\left\{A \in \mathcal{A}: A_{i, \ell+1} \notin \mathcal{A} \text { for some } 1 \leq i \leq \ell\right\} \neq \emptyset .
$$

We will show that under this assumption there exists a set $\mathcal{B} \in \mathcal{I}(n, t)$ with $|\mathcal{B}|>|\mathcal{A}|=M(n, t)$, which is a contradiction. 
Now we recall the proof of Lemma 5 by starting from the partition of $\mathcal{A}^{\prime}$ and by repeating line by line the arguments - including case a). It is easy to verify (see also the remark after the proof of case a)), that in this case, the same conclusion holds in the unrestricted case, too. So we have to prove only case b) $i=\ell+t-i$, or $i=\frac{\ell+t}{2}$. Here necessarily $2 \mid(\ell+t)$ and hence $\ell+2 \leq n$ by the assumption $2 \mid(n+t)$ and $\ell<n$. Therefore, if $\mathcal{A}\left(\frac{\ell+t}{2}\right) \neq \emptyset$, then also $\mathcal{A}^{*}\left(\frac{\ell+t}{2}\right) \neq \emptyset($ see $(3.4))$.

We have

$$
\left|\mathcal{A}\left(\frac{\ell+t}{2}\right)\right|=\left(\begin{array}{c}
\ell \\
\frac{\ell+t}{2}
\end{array}\right) \cdot\left|\mathcal{A}^{*}\left(\frac{\ell+t}{2}\right)\right| \quad(\operatorname{see}(3.3))
$$

and any $A \in \mathcal{A}\left(\frac{\ell+t}{2}\right)$ can be written in the form

$$
A=B \dot{\cup} C,
$$

where

$$
B=(A \cap[1, \ell]) \in\left(\begin{array}{c}
{[\ell]} \\
\frac{\ell+t}{2}
\end{array}\right), C=(A \cap[\ell+2, n]) \in \mathcal{A}^{*}\left(\frac{\ell+t}{2}\right) .
$$

We observe that for every $C \in \mathcal{A}^{*}\left(\frac{\ell+t}{2}\right)$ there is another element $C^{\prime} \in \mathcal{A}^{*}\left(\frac{\ell+t}{2}\right)$ with $C \cap C^{\prime}=\emptyset$. Indeed, if the element $C$ intersects with all elements of $\mathcal{A}^{*}\left(\frac{\ell+t}{2}\right)$, then any element $A_{1} \in 2^{[n]}$ of the form

$$
A_{1}=B_{1} \cup\{\ell+1\} \cup C \text { with } B_{1} \in\left(\begin{array}{c}
{[\ell]} \\
\frac{\ell+t}{2}-1
\end{array}\right),
$$

which is not in the set $\mathcal{A}$, by Lemma 2 (ii), can be added to the set $\mathcal{A}$ without violating the $t$-intersection property. This contradicts the maximality of $\mathcal{A}$.

Hence, for every $C \in \mathcal{A}^{*}\left(\frac{\ell+t}{2}\right)$, there is a $C^{\prime} \in \mathcal{A}^{*}\left(\frac{\ell+t}{2}\right)$ with $C^{\prime} \subset[\ell+2, n] \backslash C$. Since $\mathcal{A}$ is an upset and

$$
\left(B \cup C^{\prime}\right) \in \mathcal{A}\left(\frac{t+\ell}{2}\right) \subset \mathcal{A}^{\prime} \subset \mathcal{A} \text { for all } B \subset[1, \ell],|B|=\frac{\ell+t}{2},
$$

we have clearly $(B \cup([\ell+2, n] \backslash C)) \in \mathcal{A}$. It is easy to see that $(B \cup([\ell+2, n] \backslash C)) \in$ $\mathcal{A}\left(\frac{\ell+t}{2}\right)$ as well. Hence

$$
[\ell+2, n] \backslash C \in \mathcal{A}^{*}\left(\frac{t+\ell}{2}\right) \text { for all } C \in \mathcal{A}^{*}\left(\frac{t+\ell}{2}\right) .
$$


Let $\mathcal{D} \subset \mathcal{A}^{*}\left(\frac{t+\ell}{2}\right)$ be any maximal (saturated) intersecting system. From Lemma 3 we have

$$
|\mathcal{D}|=\frac{\left|\mathcal{A}^{*}\left(\frac{t+\ell}{2}\right)\right|}{2}
$$

Now consider the partition

$$
\mathcal{A}\left(\frac{\ell+t}{2}\right)=\mathcal{A}_{1}\left(\frac{\ell+t}{2}\right) \dot{\cup} \mathcal{A}_{2}\left(\frac{\ell+t}{2}\right),
$$

where

$$
\mathcal{A}_{1}\left(\frac{\ell+t}{2}\right)=\left\{A \in \mathcal{A}\left(\frac{\ell+t}{2}\right):(A \cap[\ell+2, n]) \in \mathcal{D}\right\} .
$$

We have

$$
\left|\mathcal{A}_{1}\left(\frac{\ell+t}{2}\right)\right|=\left|\mathcal{A}_{2}\left(\frac{\ell+t}{2}\right)\right|=\frac{\left|\mathcal{A}\left(\frac{\ell+t}{2}\right)\right|}{2}=\left(\begin{array}{c}
\ell \\
\frac{\ell+t}{2}
\end{array}\right) \cdot \frac{\left|\mathcal{A}^{*}\left(\frac{\ell+t}{2}\right)\right|}{2}=\left(\begin{array}{c}
\ell \\
\frac{\ell+t}{2}
\end{array}\right) \cdot|\mathcal{D}| .
$$

Finally, we consider the set

$$
\mathcal{H}=\left(\mathcal{A} \backslash \mathcal{A}_{2}\left(\frac{\ell+t}{2}\right)\right) \cup G
$$

where

$$
G=\left\{B \in 2^{[n]}: B \cap[1, \ell] \in\left(\begin{array}{c}
\ell \\
\frac{\ell+t}{2}-1
\end{array}\right), \ell+1 \in B, \text { and }(B \cap[\ell+2, n]) \in \mathcal{D}\right\}
$$

By Lemma 2 (ii) we have $G \cap \mathcal{A}=\emptyset$ and it is easy to see that $\mathcal{H} \in \mathcal{I}(n, t)$. However, $|\mathcal{H}|>|\mathcal{A}|$, because

$$
|G|=\left(\begin{array}{c}
\ell \\
\frac{\ell+t}{2}-1
\end{array}\right) \cdot|\mathcal{D}|>\left(\begin{array}{c}
\ell \\
\frac{\ell+t}{2}
\end{array}\right) \cdot|\mathcal{D}|=\left|\mathcal{A}_{2}\left(\frac{\ell+t}{2}\right)\right|(t \geq 2) .
$$

This finishes the proof. 


\section{The second proof of Theorem Ka}

Here we show how to derive Theorem Ka from Theorem AK. We keep the meaning of $n$ as in Theorem AK and let $m$ take the role of $n$ in Theorem Ka.

According to Lemma 1 , it is sufficient to settle only the case $2 \mid(m+t)$, that is by our assumption $m=t+2 r$ for some $r \in \mathbb{N}$. We recall the definitions

$$
\mathcal{K}(t+2 r, t)=\left\{A \in 2^{[t+2 r]}:|A| \geq t+r\right\}
$$

and

$$
\mathcal{F}_{r}=\left\{B \in\left(\begin{array}{c}
{[n]} \\
k
\end{array}\right):|B \cap[1, t+2 r]| \geq t+r\right\},
$$

which can be written in the form

$$
\mathcal{F}_{r}=\left\{B \in\left(\begin{array}{c}
{[n]} \\
k
\end{array}\right):(B \cap[1, t+2 r]) \in \mathcal{K}(t+2 r, t)\right\} .
$$

We also write their cardinalities as

$$
|\mathcal{K}(t+2 r, t)|=\sum_{i=t+r}^{t+2 r}\left(\begin{array}{c}
t+2 r \\
i
\end{array}\right)
$$

and

$$
\left|\mathcal{F}_{r}\right|=\sum_{i=t+r}^{t+2 r}\left(\begin{array}{c}
t+2 r \\
i
\end{array}\right)\left(\begin{array}{c}
n-t-2 r \\
k-i
\end{array}\right)
$$

For a set $\mathcal{B} \subset 2^{[t+2 r]}$ we introduce $\mathcal{B}_{i}=\mathcal{B} \cap\left(\begin{array}{c}t+2 r] \\ i\end{array}\right)$.

Here is an immediate consequence of Theorem AK:

Corollary. Let

$$
(k-t+1)\left(2+\frac{t-1}{r+1}\right)<n<(k-t+1)\left(2+\frac{t-1}{r}\right),
$$

then

$$
\max _{\mathcal{B} \in \mathcal{I}(t+2 r, t)} \sum_{i=t}^{t+2 r}\left|\mathcal{B}_{i}\right| \cdot\left(\begin{array}{c}
n-t-2 r \\
k-i
\end{array}\right)=M(n, k, t),
$$

and the maximum is assumed at the unique set $\mathcal{B}=\mathcal{K}(t+2 r, t)$.

The following, rather obvious, "Comparison Lemma" makes it possible to prove easily Theorem Ka via the above stated Corollary. 
Comparison Lemma. Let $\alpha_{t} \geq \alpha_{t+1} \geq \ldots \geq \alpha_{t+2 r} \geq 0, \alpha_{t} \neq 0$, be a nonincreasing sequence of real numbers such that

$$
\max _{\mathcal{A} \in \mathcal{I}(t+2 r, t)} \sum_{i=t}^{t+2 r}\left|\mathcal{A}_{i}\right| \cdot \alpha_{i}
$$

is assumed at $\mathcal{A}=\mathcal{K}(t+2 r, t)$. Then the same holds, if $\alpha_{t}=\alpha_{t+1}=\ldots=\alpha_{t+2 r}=1$, i.e.

$$
\max _{\mathcal{A} \in \mathcal{I}(t+2 r, t)} \sum_{i=t}^{t+2 r}\left|\mathcal{A}_{i}\right| \cdot 1=\max _{\mathcal{A} \in \mathcal{I}(t+2 r, t)}|\mathcal{A}|=M(n, t)
$$

is assumed at $\mathcal{A}=\mathcal{K}(t+2 r, t)$, which is exactly Theorem Ka.

Proof. The result easily follows from the following fact:

If $\mathcal{B} \neq \mathcal{K}(t+2 r, t), \mathcal{B} \in \mathcal{I}(n, t)$, and $\sum_{i=t}^{t+2 r}\left|\mathcal{B}_{i}\right| \cdot 1>\sum_{i=t}^{t+2 r}\left|\mathcal{K}_{i}(t+2 r, t)\right|=\sum_{i=t+r}^{t+2 r}\left(\begin{array}{c}t+2 r \\ i\end{array}\right)$, then, since $\alpha_{t} \geq \alpha_{t+1} \geq \ldots \geq \alpha_{t+2 r} \geq 0$, we would have

$$
\sum_{i=t}^{t+2 r}\left|\mathcal{B}_{i}\right| \cdot \alpha_{i}>\sum_{i=t}^{t+2 r}\left|\mathcal{K}_{i}(t+2 r, t)\right| \cdot \alpha_{i}
$$

This contradicts the assumption in the Lemma.

Remark. The "Comparison Lemma" can be formulated in a more general form by assuming weaker conditions on $\alpha_{t}, \alpha_{t+1}, \ldots, \alpha_{t+2 r}$, and the conclusion (even in more general form) of the Lemma still holds. However, this is not needed here.

Proof of Theorem Ka. According to the corollary and the Comparison Lemma, it is sufficient to show the existence of integers $k, n$ (for fixed $t, r$ ), such that both

$$
\begin{aligned}
& (k-t+1)\left(2+\frac{t-1}{r+1}\right)<n<(k-t+1)\left(2+\frac{t-1}{r}\right) \text { and } \\
& \left(\begin{array}{c}
n-t-2 r \\
k-t
\end{array}\right) \geq\left(\begin{array}{c}
n-t-2 r \\
k-t-1
\end{array}\right) \geq \ldots \geq\left(\begin{array}{c}
n-t-2 r \\
k-t-2 r
\end{array}\right) \text { hold. }
\end{aligned}
$$

A simple calculation shows, that this is the case for any $k, k>\frac{(t+2 r-1) r}{t-1}+t$, and $n$ as the biggest integer from the interval given by the first inequality. For these $k$ and $n$ the second inequality holds. Let us note that for $k>\frac{(t+2 r-1) r}{t-1}+t$ we have

$$
(k-t+1)\left(2+\frac{t-1}{r}\right)-(k-t+1)\left(2+\frac{t-1}{r+1}\right)>1,
$$

which guarantees the existence of an integer $n$. 


\section{References}

[1] R. Ahlswede and L. H. Khachatrian: The complete intersection theorem for systems of finite sets, Europ. J. Combinatorics, 18 (1997), 125-136.

[2] P. ERdős, C. Ko, and R. RAdo: Intersection theorems for systems of finite sets, Quart. J. Math. Oxford Ser., 12 (1961), 313-320.

[3] G. O. H. Katona: Intersection theorems for systems of finite sets, Acta Math. Acad. Sci. Hungar., 15 (1964), 329-337.

Rudolf Ahlswede

Universität Bielefeld

Fakultät für Mathematik

Postfach 100131

33501 Bielefeld

Germany
Levon H. Khachatrian

Universität Bielefeld

Fakultät für Mathematik

Postfach 100131

33501 Bielefeld

Germany

lk@mathematik.uni-bielefeld.de 